\title{
Viewpoints on Microscopic and Mesoscopic Transformation Processes in Martensite
}

\author{
J.A. Krumbansl \\ University of Massachusetts, Amherst MA 01002, U.S.A.
}

\begin{abstract}
The purpose of this presentation is to provide some perspective gained from recent studies in condensed matter physics on the microscopic processes by which martensitic transformations take place to supplement the highly developed crystallographic and thermodynamic descriptions. Atomic scale dynamics can be related to mesoscale pattern formation, in idealized systems, and the energies of twin boundaries and ferroelastic patterns can be computed from such data, at least in principle. An overview of the formalism used utilizing nonlinear and strain-gradient elasticity is given. Yet in reality probably all transformations of laboratory or manufactured materials are controlled by defects. An approach to the defect situation based on the framework developed for the idealized martensite is given. Finally a few remarks are made relating this general viewpoint to shape memory materials.
\end{abstract}

\section{INTRODUCTION.}

It would be incorrect to think of this report as that of a general theory of martensite; the magnificent edifice that has been constructed over the past century with scientific and practical leadership by such innovative scientists as Bain, Cohen, Christian, Read, and their disciples will continue to stand as the framework into which most of the major developed features of the observations on martensitic materials can be placed. At the same time the factors underlying the major steps in the transformation process, such as nucleation, elastic softening, precursors, etc., remain open topics in many respects. Indeed, the great new interests in so-called smart materials, e.g. shape memory materials, call for deeper understanding and control of those phenomena which occur in processing.Meanwhile new theories and experimental methods have achieved in physics much new insight over the past two decades. It is in the spirit of supplementing the great amount that is known traditionally in this subject with insights from more recent experimental results and theoretical developments in condensed matter physics that this report is written.

In addition to the developments in condensed matter physics, it is also worth pointing out the fact that today a number of other disciplines have been brought to bear on features of martensite at three different scales, as suggested in Fig. 1, from the nanoscale to micron to macroscopic dimensions. We can consider that in a very real sense the study of martensite can be an ecumenical teacher of materials science.

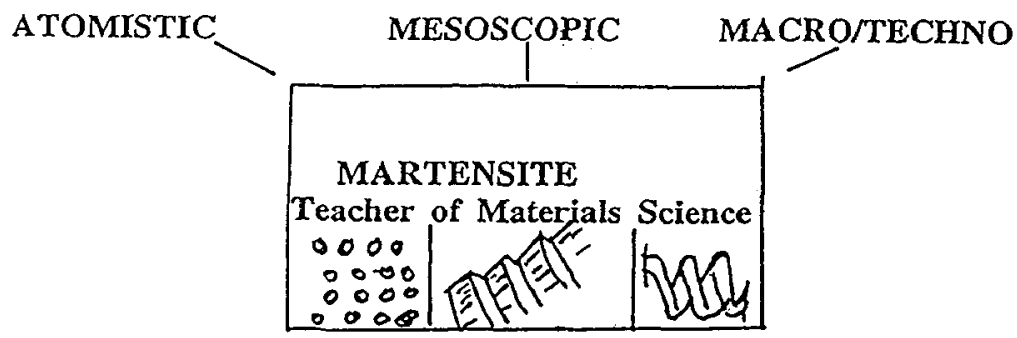

Figure 1. A Portrait of Martensite. Three scales: nanometers, microns, centimeters. 
At the atomistic scale several areas of basic science relate: C.M. Physics, S.S. Chemistry, Metallurgy, Ceramics, Minerology, at least [1-4]. At the mesocopic scale there is activity in : Continuum Mechanics, Dislocation Mechanics, and recently much activity in Modern Applied Mathematics [5]. And at the macroscopic and engineering scales it is particularly interesting to see the functional developments based on the properties of martensitic materials: Shape Memory Materials [6], Transformation Toughening [7], and others. There are some phenomena that span the whole set of scales, such as the studies of hysterisis and its origin in stress induced transformations, training, and so on.

In view of this multifaceted nature of martensite research it is often difficult to communicate in this field since so many disciplines are involved and research objectives differ. Indeed, it is almost impossible to find a single generally accepted definition of martensite, so it is appropriate that we define the territory which we focus on here. We choose to circumscribe our class of martensitic materials as those in which transformations are purely displacive, with a multiplicity of symmetry connected product variants from a higher symmetry parent, which are formed in a solid-solid transition, thus placing coherency stresses on the products as they form, yielding arrays of the variant which minimize the overall balance between internal, intervariant boundary, and product-parent habit plane free energies. Many martensitic materials qualify as having these properties. Even within this definition there seem to be three distiinct subclasses: Strongly Distortive, transformation strain $\mathrm{e}=O\left(10^{-1}\right)$, e.g. Fe-C, steels, where generally coherency stress is relieved by plastic flow in the form of slip bands, and lack of easy shape reversibility; Moderately Displacive, $e=O\left(2-5 \times 10^{-2}\right)$, shape memory alloys, definitely first-order transformations; Weakly Displacive, e.g. the A-15 super- conductors, and other essentially second order transforming materials. The objective here is mostly to connect the atomistic scale physics with the continuum mechanics level. This is not easily done because of topological disconnects at the atomic scale from plastic flow in the strong distortion martensite; and at the other end of the nearly second order transitions, critical fluctuations require non-analytic free energy function(al)s.However, many of the Moderately Displacive shape memory materials do nearly conform to the subclass of topologically, elastically reversible transformations and thus a reasonable connection can be made between the atomistic and mesoscopic levels; we focus on the physics of these.

\section{MODELING AN IDEAL INTRINSIC MARTENSITIC MATERIAL.}

\subsection{Spatially Uniform Phases}

We report here developments in modeling the martensite transformation and mesoscopic structure of a homogeneous, nominally defect free material like the moderately displacive alloys FePd, InTI, Ni Al, and some of the $\mathrm{Cu}$ ternary alloys. It turns out that the framework developed through this research provides a way in principle of thinking about the general basic phenomenology of the transformation process; though in actuality, as we discuss in the Section 3 it seems likely that defects of one sort or another dominate all real martensitic transformations. The approach and detailed formalism is discussed in ICOMAT-92 by G. R. Barsch and the writer.(BK) The rationale is well put by John Cahn [3]: "Scientific studies often begin with the simplest possible aspects of a phenomenon. Subsequently, the knowledge gained from the study of simple examples is combined to tackle ever more complex problems."

The behaviors we wish to connect are: 1)Transformations are purely distortive not involving compositional or diffusive changes, the extrinsic thermodynamic variables are strains or "shuffles" only; 2) We consider only those transformations which are topologically reversible at both the macroscopic and lattice scales; that is, a lattice correspondence by continuous atomic displacements exists, which implies that the symmetry group of the product phase is a subgroup of the parent phase. (This does not require the transition to be second order, though those satisfy this requirement.); 3 ) The structure of the intertwin and parent-product interfaces. Here we consider only the case where temperature $T$ is the intrinsic parameter.

In this situation the parent and product states for the uniform phases are connected via a free energy function which involves the (complex) amplitudes of the lattice strain and intracell atomic rearrangements, i.e." distortive order parameters" that make the correspondence between the parent and product phases. This function has multiple minima in the space of its order parameters; since we discuss first order transitions critical fluctuations are negligible so the free energy can be quite well represented by a (nonconvex) polynomial function, with $\mathrm{T}$-dependent parameters. This is not an arbitrary method; Landau pointed out that in principle one could find a "mean field" free energy by computing partition functions, with the macroscopic order parameters constrained to take on certain values, from which the free energy could be found. The procedure is widely used in ferroelectrics and ferromagnetics, under the rubric of "Landau Free Energy".

It is desired to describe the lattice correspondence in terms of the Bain i.e. elastic strain, and the intracell shuffle displacements which carry the parent lattice into variants of the product lattice, nominally "austenite to martensite" as a generic term, even though the material in question is not at all ferrous. One should really think of this lattiice distortive transformation in general terms, not simply as an elastic one. 
Though usually constructed for small amplitude lattice displacements the conventional elastic strains and phonon normal modes constitute a complete basis set for representing distortions that connect the parent and product phases, the former for the Bain strain, the latter the intracell shuffle displacements. In particular it is efficient to choose symmetry appropriate combinations of the cartesian elastic strains and the phonon modes; the set of these can be denoted $\left\{\mathrm{e}_{\alpha}\right\}$ and $\left\{\varphi_{\beta}\right\}$, or e and $\varphi$ for short; usually, $(\mathbf{e}, \varphi)=0$ in the parent phase. These are referred to as "order parameters". The appropriate choices for cubictetragonal, and tetragonal-rhombohedral are discussed by BK. While most martensitic transformations can be described in this way symmetry theory also can demonstrate that in some cases the direct transformaion is not allowed by continuous distortions and one must consider intermediate crystalline phases $[8,9]$. None the less, pedagogically it has proven informative to consider models in which the direct transformation is allowed; many shape memory alloys fall in this class. As will also be seen this procedure allows the connection bewtween atomic motions and continuum mechanics. To illustrate how a mixed strain(frozen)phonon description of the distortion of a parent phase can yield various product phases, refer to Fig. 2 and Table 1 below.

TABLE 1 Classification of the b.c.c.-based martensites by the phonon description

\begin{tabular}{|c|c|c|c|c|c|c|c|c|}
\hline \multirow[t]{2}{*}{ Parent } & \multirow{2}{*}{\multicolumn{2}{|c|}{ Martensite }} & \multirow{2}{*}{$\begin{array}{l}\text { Latrice } \\
\text { symmetry }\end{array}$} & \multicolumn{2}{|c|}{ Phonon made } & \multirow[t]{2}{*}{$\varphi(r)$} & \multirow[t]{2}{*}{ Slip $\bar{\varepsilon}_{2}$} & \multirow[t]{2}{*}{ Examples } \\
\hline & & & & $q_{11}$ & $m$ & & & \\
\hline \multirow[t]{2}{*}{ b.c.c. } & $\begin{array}{l}2 \mathrm{H} \\
4 \mathrm{H} \\
6 \mathrm{H} \\
7 \mathrm{R} \\
9 \mathrm{R}\end{array}$ & $\begin{array}{c}1 \overline{1} \\
2 \overline{2} \\
3 \tilde{3} \\
5 \overline{2} \\
(2 \overline{1})_{3}\end{array}$ & $\begin{array}{l}\text { Hex. } \\
\text { Ortho. } \\
\text { Ortho. } \\
\text { Ortho. } \\
\text { Ortho. }\end{array}$ & 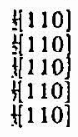 & $\begin{array}{l}1 q \\
1 q \\
1 q \\
1 q\end{array}$ & $\begin{array}{l}0 \\
120^{\circ} / 2 \\
-60^{\circ} / 3 \\
0 \\
0\end{array}$ & $\begin{array}{l}0 \\
\pm 1 / 12 / 2 \\
\pm 1 / 12 / 3 \\
1 / 8 / 7 \\
1 / 6 / 3\end{array}$ & $\begin{array}{l}\mathrm{Zr}, \mathrm{Li}, \mathrm{Mg}, \mathrm{Na}, \mathrm{CuAlNi}, \mathrm{CuSn}, \mathrm{AgAl}, \mathrm{AuCd} \\
\mathrm{CuSn} \\
\mathrm{NiAl} \\
\mathrm{Li}, \mathrm{CuAl}, \mathrm{CuSn}, \mathrm{CuZn}, \mathrm{CuPd}, \mathrm{AgCd}, \mathrm{AuCuZn} n_{2}, \mathrm{AuO}\end{array}$ \\
\hline & $\begin{array}{l}\zeta \\
\omega \\
\gamma\end{array}$ & & $\begin{array}{l}\text { Rhombo. } \\
\text { Rhombo. } \\
\text { Cubic }\end{array}$ & 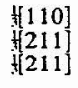 & $\begin{array}{l}3 q \\
3 q \\
12 q\end{array}$ & $\begin{array}{l}0 \\
0 \\
0\end{array}$ & $\begin{array}{l}0 \\
0 \\
0\end{array}$ & $\begin{array}{l}\text { AgZn, AuCd, TiNi } \\
\text { Zr, NbZr, TiMo } \\
\text { CuZn, AiZn }\end{array}$ \\
\hline f.c.c. & $\begin{array}{l}\text { f.c.t. } \\
\text { f.c.o. }\end{array}$ & & $\begin{array}{l}\text { Tetra. } \\
\text { Ortho. }\end{array}$ & $\begin{array}{l}-0 \\
-0\end{array}$ & $\begin{array}{l}1 \\
2\end{array}$ & $\begin{array}{l}0 \\
0\end{array}$ & $\begin{array}{l}0 \\
0\end{array}$ & $\begin{array}{l}\text { FePd, InTl } \\
\text { NiMo }\end{array}$ \\
\hline
\end{tabular}

The static displacement $u(r)$ in the martensite phase is generally expressed by

$$
u(r)=\sum_{x=1}^{\prime \prime}\left\{\xi e\left(q_{0}{ }^{*}\right) \cdot r+\varphi(r)+c \cdot c\right\}+e_{2} \cdot r+e_{3} \cdot r
$$

where $e\left(q_{0}{ }^{x}\right)$ is the phonon eigenvector, $m$ is the multiplicity of the equivalent modes, $\varphi(r)$ is the phase factor, $e_{3}$ is the deviatoric strain and $e_{2}$ is the shear strain.

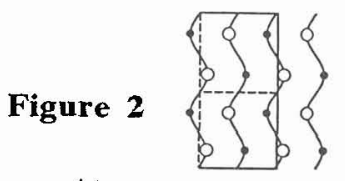

(a)

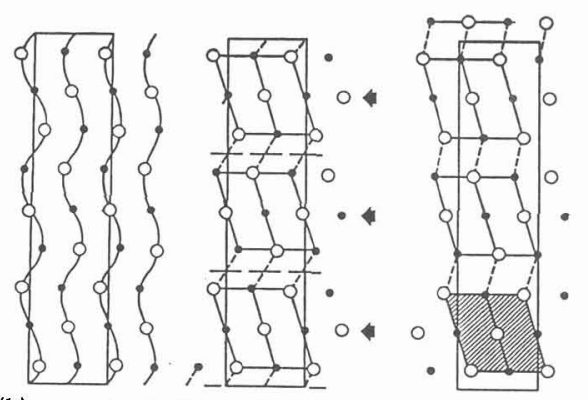

(b)

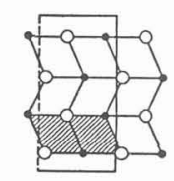

Yamada
Phonon description of the important martensite structures: (a) $2 \mathrm{H}$ structure-this structure is considered to be constructed by the "condensation" of the phonon mode

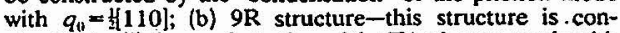
structed by (i) the condensation of the TA phonon mode with $q_{\mathrm{a}}=\mathbf{y}[110]$ and (ii) additional uniform shear strain indicated by the thick arrows.

In this manner the Landau free energy for displacive transitions can be written in a form depending on strain, shuffles, and coupling between them; it is to be considered as a generalized, nolinear elastic free energy. If the functions are purely quadratic, and shuffles are neglected it reduces to conventional 
elasticity. The functions can be expressed as polynomial (i.e.Taylor) expansions about the parent phase with temperature dependent coefficients. If one distortive order parameter $\Phi$ dominates the transition protypical Landau forms for first and second order transitions are shown in Fig.3.The case where both shuffles and elastic strain must be considered is discussed by Lindgòrd and others[10].

Figure 3. The two prototype forms of the Landau free energy.

(a) Second order transition, equation (7);

(b) Different regimes for a first order transformation, equation (6): $a=3 / 16$ corresponds to the transformation temperature.
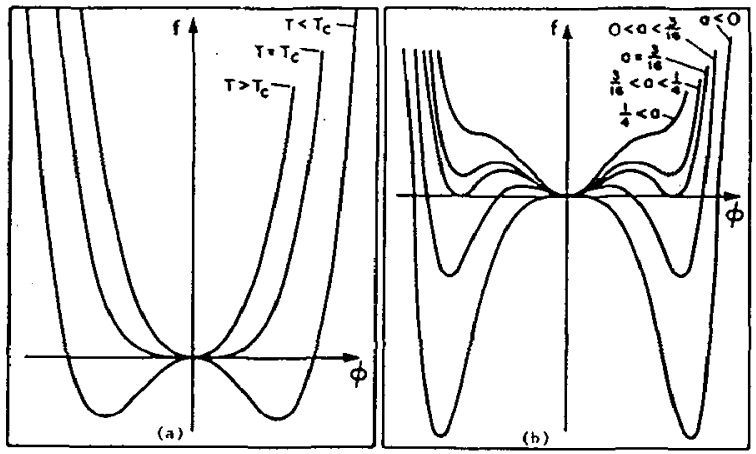

A very important observation to make on the difference between the first and second order cases is as follows: if the system is in a stable or metastable phase it is at a local minimum of free energy; for small oscillations about this configuration the generalized elastic stiffness is proportional to the quadratic curvature of the free energy. It is then to be noted that for second order transitions that curvature and the restoring force go to zero at the transition condition i.e. that distortive mode softens; for first order transitions, however, this dynamic ( i.e.soft-mode )instability does not occur. First order martensitic transitions are not driven simply by soft modes and it is misguided to look just to them.[10],[11].In general the control parameters for first order transitions are combinations of the several coefficients in the anharmonic Landau expansion.Fig.4 compares the phonon spectrum for a second order transition in $\mathrm{SrTiO}_{3}$ with the first order transition in the specified NiAl alloy; if the latter transition, B-cubic to $7 R(M)$ were to take place by a soft mode mechanism it would be at about minus $40 \mathrm{~K}$; in fact the martensitic transition occurs at circa $+80 \mathrm{~K}$.

Figure 4.
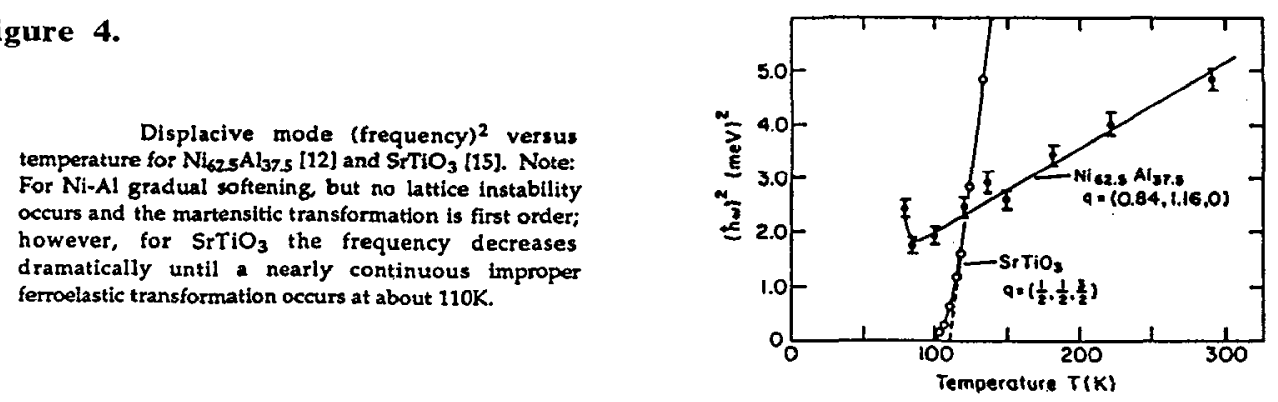

In fact the NiAl transition involves a coupling of a transverse mode at $q \sim 0.16$ along [110] with a shear elastic mode, for which the theory has yet to be developed quantitatively. 
The generic nature of a first order proper ferroelastic transition is discussed by BK for a purely elastic cubic-tetragonal transition. The distortive order parameter has two components, $e_{2}=\left(e_{11}-e_{22}\right) / \sqrt{3}$ and $e_{3}$ $=\left(\mathrm{e}_{11}+\mathrm{e}_{22}-2 \mathrm{e} 33\right) \sqrt{ } / 2$ which are recognized as purely deviatoric strains. In higher order there are couplings to other strains but the leading terms in the nonlinear "elastic" free energy $\Phi_{L}$ for the cubictetragonal corespondence of spatially homogeneous phases is (BK)

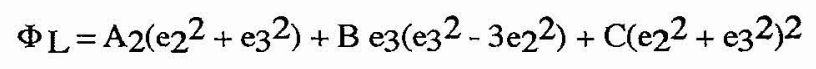

$\mathrm{A}_{2}, \mathrm{~B}, \mathrm{C}$ are temperature dependent second, third, and fourth order elastic constants respectively.If in particular (but not in general) $A_{2} \sim a\left(T-T_{C}\right)$ where $T_{C}$ is the hypothetical soft mode temperature $\quad(-40 \mathrm{~K}$ in $\mathrm{NiAl}$ ) the first order transition temperature is $\mathrm{T}_{1}=\mathrm{T}_{\mathrm{c}}+\mathrm{B}^{2 / 4 a C}$, which, assuming (a, C) positive as they are in anharmonic theories, means that the actual transition temperature is both higher than the soft mode instability temperalure and strongly dependent on the higher order elastic constants. Indeed it is apparent that first order transitions can occur quite without mode softening, determined only by higher order anharmonicity. In the Fig.5 below is shown the schematic free energy for the cubic-tetragonal transition, as developed by BK. The parameter $\tau=\left(T-T_{c}\right) /\left(T_{1}-T_{c}\right)$ is a dimensionless temperature; $\mathrm{T}_{1}$ is the first order transition temperature and $\mathrm{T}_{\mathrm{c}}$ is the hypothetical instability temperature. The figure caption discusses the (approximate) trajectories in free energy space to go between two variants (twin boundary) and between the cubic and one tetragonal variant.The transition region is a static "soliton" functionally.
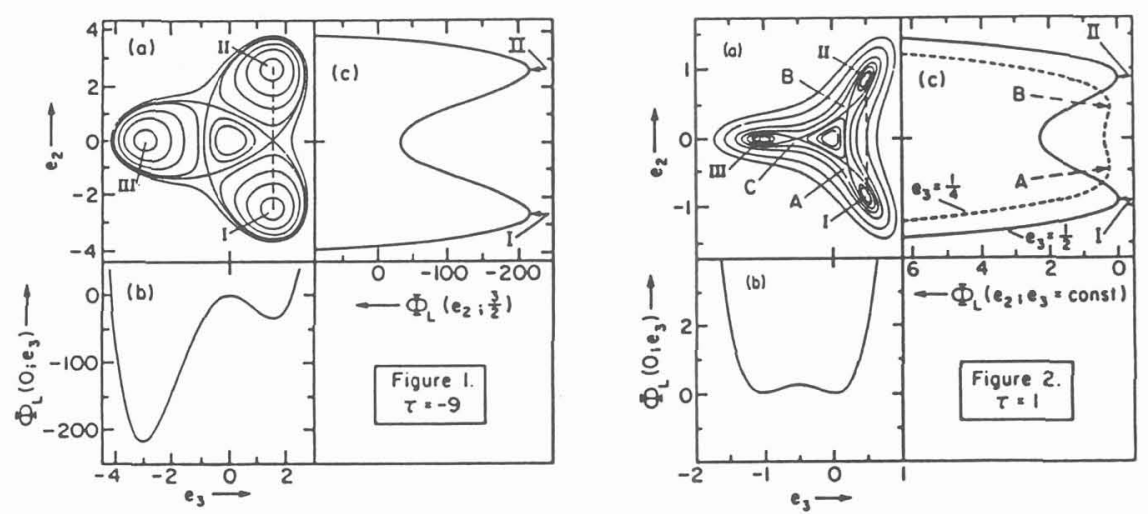

Figure 5. Nonlinear elastic energy $Q_{L}\left(e_{2}, e_{3}\right)$ as function of the deviatoric atrains ezez for $\tau=-9$ and $\tau=1$,

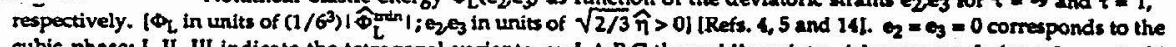
cubic phase; I, II, III indicate the tetragonal variants, and A,B,C the saddle points. (a) contours $\Phi_{L}$ (e2 ez)-const; (b) sections e2=0; (e) rections e3mconst that contain either the minima I and II, or the saddle points $A$ and $B$. The double well potentials in Figs. $1 \mathrm{c}$ and $2 \mathrm{c}$ show the energy barrier that must be surmounted when joining two different tetragonal domains I and II (or pretransformation orlentation states $A$ and $B$ ) by ferroelastic domain walls. Note: Only for $A_{2}^{\circ}<<$ $\left[2 A_{1}+(3 / 2) A_{3}\right]$ is the strain profile $\left(e_{2}(s)\right.$, ez $\left.(s)\right]$ for $\tau *-9$ given by either of the two paths $I-J I$, or $A-B$, in Figs. $2 a$ and $2 c$ for twin boundaries and pretransformation strain modulations, respectively.

\subsection{Nonuniform strain; distortive phase boundaries; twinning.}

Having developed a formalism for describing phenomenologically the transformation of one homogeneous phase into another structural phase purely displacively, in continuous fashion. In fact, this is not the way solid-solid transformations take place. In martensite the strain is far from uniform, but varies rapidly in space both in the regions of the habit plane, and multivariant interfaces i.e twin boundaries in a twinning martensite. The twinning martensites probably include most shape memory materrials and A-15s which show martensite structures. The strong martensites e.g. ferrous which relieve coherency stress by plastic flow and slip band development are not covered by this formalism. Because the Landau formalism itself for uniform bulk phases does not contain terms in spatial derivatives of the (strain) order parameters it does not have the capability of describing strain variations over short distances e.g. $O$ (nanometer). There are two approaches for proceeding: 1) If it is known that the interphase boundaries are no more than a few atomic layers in extent lattice simulations are probably the most convenient approach; 
2) If however, there is any indication that the boundary (including its elastic strain field) extend over several lattice constants or more, unless very large computational capacity is available and thoughtful program design/data presentation are employed in a lattice simulation elastic effects will be missed.

Instead, some time ago extensions of simple elasticity theory to include strain-gradients SG were developed [12]; while apparently quite little known in the materials and physics community they have been widely explored in the applied mathematics and theoretical mechanics field, in liquid crystals [13], ferroelectrics [14] ; in superfluids Ginzburg introduced the gradient of the order parameter to augment Landau theory, henceforth "Landau-Ginzburg" [15].

Thus, "curvature elasticity" is the next order beyond homogeneous strain elasticity formalism, and so forth if higher order gradients are necessary. We applied that method, since for several representative shape memory SM materials experimental studies [3] established that twin boundaries and habit planes are at least several layers thick, as we shall see now. Before proceeding, we note that criticisms have been expressed of the validity of the strain-gradient extension of elasticity i.e. "why stop at simply adding SG," why not higher gradients; and after all, these are only approximations to the real phonon representations." The problem with this non-quantitative complaint is that the same criticism applies to the use of conventional elasticity theory for situations where the strain varies at all in space, "after all elasticity theory is not valid except at infinite wavelength"!. The reply is straightforward: indeed all elasticity theories are incorrect in principle for inhomogeneous strain in a real crystal. If, however, a Fourier analysis shows that the only important spectral components of the strain field involve wave numbers very much smaller than a reciprocal lattice constant the criticism that a particular representation doesn't reproduce the phonon dispersion relation out to the zone boundary is quite irrelevant. In the application to twin boundaries, habit planes, and defect problems which we have studied in twinning martensites the characteristic length scales are such that the dominant strain field Fourier components are limited to $\sim 20 \%$ of the Brillouin zone. Then, examining the phonon dispersion curves quantitatively, BK concluded that in the materials considered the addition of the first order strain gradient to uniform strain elasticity suffices to provide an accurate correction for the nonuniform strain fields under consideration. The detailed development of strain gradient theory in this context is beyond the scope of this paper, and it is given in some detail by the second of the two BK papers in ICOMAT-92. However many of the main results are easy to understand.

One of the most characteristic features is that for small amplitude phonons in a number (not all) of martensitic materialds the deformation mode dispersion relation for small $\mathbf{k}$ is fitted by

$$
\rho_{0} \omega_{\lambda}^{2}(k)=c_{\lambda} k^{2}+d_{\lambda} k^{4}+\ldots .
$$

where $c_{\lambda}$ is the velocity squared of very long wave sound, proportional to the appropriate ordinary elastic modulus, while $d_{\lambda}$ is a linear combination of the strain gradient SG coefficients. In other words the strain gradient terms introduce dispersion, the lowest order correction to simple elasticity theory, attempting to correct for the longer wave appearance of phonon dispersion. In BK [2] there is detailed discussion of how the SG coefficients can be related to experimental neutron scattering data. The procedure for determining the defining equations for displacement fields then parallels the case for ordinary elasticity except that now the Lagrangian is a functional of the local kinetic energy density, nonlinear in the strains and strain gradients; in general "nonlinear, nonlocal", for spatially varying strain. The application of the Euler-Lagrange method then yields both the static and dynamic defining equations. They are generally solvable in closed form for only special cases of the parameters. A full development for cubic-tetragonal transitions is given by BK ICOMAT 92.

However, there are two interesting features of note in this formalism. First, if $d_{\lambda}$ is positive the phonon dispersion curvature is upward as $\mathrm{k} \rightarrow 0$, and the ultrasonically determined dispersionless elastic wave frequency line lies below the strain gradient model ( or in the lower k-region, below experimental phonon frequency ) This is found for many SM twinning martensites, but definitely not for some other materials, the alkali metals Li andNa notably. The point is that if the SG coefficient is positive then it is possible to find continuous, stable twin boundary transitional solutions connecting variants. (Fig.6 ) Note: 1) Dislocations are not necessary at all.;2) The transition region spreads over many lattice constants. 3) (in this defect free model crystal) To move this domain wall requires no vacancy motion so such a boundary can be expected to move reversibly and in glissile manner with speed approaching that of sound It would move with the character of a soliton, not with dislocation kinematics. It seems reasonable to also suggest that for these materials the parent-product interface might move similarly. All of these properties are ideally suited to shape memory applications. However, we will see below, under "DEFECTS", that they also provide models for precursors and athermal nucleation. 
Figure 6. $\quad(1 \overline{1} 0)$ twin boundary between two tetragonal variants found from nonlinear, nonlocal continuum elasticity; note the absence of dislocations [4]. $\left[\mathrm{e}_{2}^{\infty}=(3 / \sqrt{2}) \hat{n}=0.14\right.$.

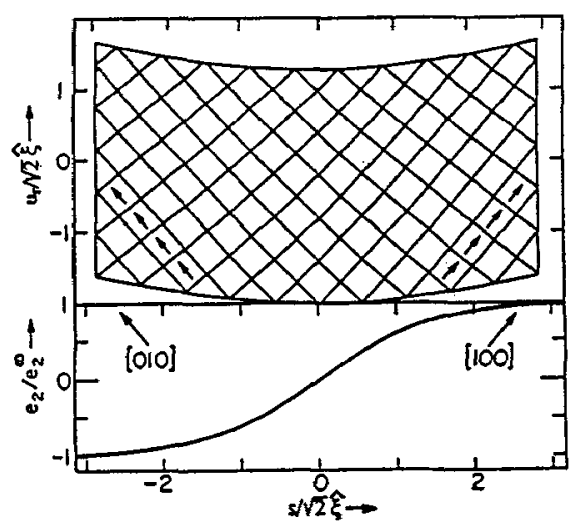

A second feature can be gleaned from the dispersion relation; rewrite it in the form

$$
\rho_{0} \omega_{\lambda}{ }^{2}=\mathrm{c}_{\lambda} \mathrm{k}^{2}\left[1+\left(\mathrm{d}_{\lambda} / \mathrm{c}_{\lambda}\right) \mathrm{k}^{2}\right]
$$

and it is seen that $\left(d_{\lambda} / c_{\lambda}\right)$ is a characteristic length-squared. In essence then, the criterion that the SG correction can be ignored is that the strain varies significantly only over distances large compared to this characteristic length. As to whether higher order SG terms must be considered or not, analogous criteria can be developed. For our present applications the first order SG correction suffices. Though that point needs verification in specific cases; the procedure is clear.

For high symmetry directions the SG parameters, e.g. $d_{\lambda}$, can be determined from the phonon dispersion curves, at least in principle. BK in ICOMAT-92 used the neutron data for Fe-Pd and an In-TI alloy for that purpose. The dispersion curves along the [110] direction for the shear mode involved in the martensitic transformation in those materials are shown in Fig.7 [16,17]. It is quite apparent that in the FePd alloy the curvature is upward so the SG coefficient $d_{S}$ is positive, and we expect that curvature elasticity contributions, continuous and dislocation free twin boundaries, etc. will be found. A number of other shape memory alloys also show dispersion curves rising above ultrasonically determined long wave slopes should also come within the scope of the theory above. At the same time the martensitic alkali metals $\mathrm{Li}$ and $\mathrm{Na}$ seem to have clear negative $\mathrm{d}_{\mathrm{s}}[18,19]$ for which the twinning soliton model of an intervariant boundary is unstable; in these cases (c.f. Clapp, [19]) it is essentially certain that undercooling and defect nucleation of the transition is required.
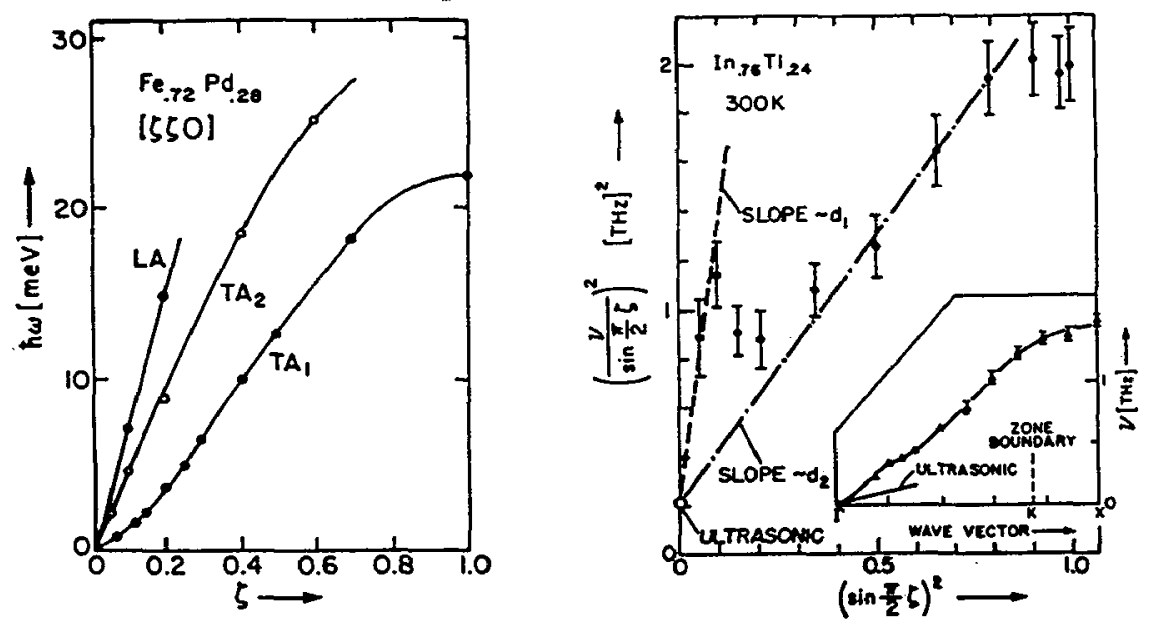

Figure 7. Phonon dispersion for Fe. $72 \mathrm{Pd} .28$, and $\mathrm{In} .76 \mathrm{Tl} .24$ at room temperature. The upward curvature for FePd is apparent; the situation for InTl has greater experimental uncertainty. 
2.3 Summary and Perspective, Defect Free Model.

The formalism we have reviewed in the preceding sections is put into comparative perspective in Fig. 8 below.

\begin{tabular}{|c|c|c|c|c|c|}
\hline Defect & $\begin{array}{l}\text { Topological } \\
\text { discontinuity }\end{array}$ & $\begin{array}{l}\text { Core Region } \\
\text { Atomistic theory } \\
\text { (model dependent) }\end{array}$ & $\begin{array}{l}\text { Long-Range Field } \\
\text { Continuum theory } \\
\text { (generic) }\end{array}$ & & \\
\hline \multirow[t]{3}{*}{ Dislocations } & \multirow[t]{3}{*}{ Burgers Vector } & \multirow{3}{*}{$\begin{array}{l}\text { Frenkel and } \\
\text { Kontorova (Ref 50) } \\
\text { Peierls (Ref 5T) }\end{array}$} & & $\begin{array}{l}\text { Local } \\
\text { elasticity theory }\end{array}$ & $\begin{array}{l}\text { Nonlocal } \\
\text { elasticity theory }\end{array}$ \\
\hline & & & $\begin{array}{l}\text { Linear } \\
\text { theory }\end{array}$ & $\begin{array}{l}\text { Volterra (Ref 69) } \\
\text { Eshelby (Ref 52) }\end{array}$ & Eringen (Ref 72) \\
\hline & & & $\begin{array}{l}\text { Nonlinear } \\
\text { theory }\end{array}$ & $\begin{array}{l}\text { Seeger and } \\
\text { Mann (Ref 70) } \\
\text { Teodosiu (Ref } 71 \text { ) }\end{array}$ & \\
\hline \multirow{3}{*}{$\begin{array}{l}\text { Interfaces: } \\
\text { (ferroelastic) } \\
\text { twin } \\
\text { boundaries }\end{array}$} & \multirow{3}{*}{$\begin{array}{l}\text { Shape Change } \\
\text { Local mirror } \\
\text { plane; broken } \\
\text { translation } \\
\text { symmetry }\end{array}$} & \multirow{3}{*}{$\begin{array}{l}\text { Sutton and } \\
\text { Christian (Ref 67) } \\
\text { Sutton and } \\
\text { Vitek (Ref 68) }\end{array}$} & & Local & Nonlocal \\
\hline & & & $\begin{array}{l}\text { Linear } \\
\text { theory }\end{array}$ & $\begin{array}{l}\text { Christian (Ref 20) } \\
\text { Olson and Cohen (Ref 21) }\end{array}$ & \\
\hline & & & $\begin{array}{l}\text { Nonlinear } \\
\text { theory }\end{array}$ & & Present work \\
\hline
\end{tabular}

Figure 8. Atomistic and continuum methods for crystal defects. Symmetry breaking by either dislocations or (ferroelastic) twin boundaries. (After BK in "Martensite "Eds. G.B. Olson and W.S. Owen, ASM 1992)

The idea behind this table is to compare the methodology developed here with the more familiar history of dislocations. Dislocations and the twin boundary have in common the property that they are topological defects. The first models of dislocations, Frenkel-Kontorova and Peierls, were atomistic in character; their topological nature appeared in the form of the Burgers Vector. As the subject developed, however, it became clear that it was necessary to incorporate the fact that dislocations produced long range elastic fields.and to incorporate continuum descriptions to complement the atomistic core calculations. Indeed, for many applications the elastic continuum component dominates the physics and the core details are secondary. As the table indicates, from the initial use of linear elasticity by Eshelby the continuum methods evolved to incorporate both nonlinear and nonlocal (of which one form is strain gradient) elasticity.

In the present discussion the object of interest is, for example, a twin boundary - a planar topological defect rather than a line defect. As in the dislocation case much of the initial development has been on an atomistic basis; this has been greatly augmented as computer simulation capabilities have grown so rapidly. If, as noted earlier, the transition region is only a few atomic layers in thickness, the atomistic approach is most informative. However, if the twin boundary, or parent-variant interface are not highly localized then continuum methods are needed. Some work on nucleation, based on heuristic models which included strain gradient terms in the free energy, have been studied by Olson and Cohen [20].The systematic development by Barsch and the author of a generic methodology, in a nonlinear, nonlocal (strain-gradient) framework, based on the Landau-Ginzburg idea is what has been reported here.

Only a few demonstration applications have been worked out in some detail so far [21], but we think that this framework can be used as a base for studying the effects of defects, applied stresses, dynamics, etc., starting from the idealized crystal, and then perturbing accordingly. Much can be done in this manner, and remains to be done. In the next section we review a defect model for athermal martensitte, and a model for tweed precursors in the framework of the nonlinear, nonlocal generalized elasticity just presented. 


\section{Defects in Transforming Materials; Martensite.}

The topic of defects in solids is one of the oldest, so it may be surprising that anything new can be added, but in regard to the distortions of the crystal around a defect it seems that there is an important situation which has not been formalized in a generic way. The circumstances are as follows. First, consider the conventional case, for example when a larger/smaller atom is substituted for the normal atom in a crystal. The resulting distortions of the lattice are treated either by continuum elasticity (Eshelby and others), or by the Kanzaki method. In either case the host crystal is not considered to change phase; its elastic response, or phonon Green's function, is that of the unperturbed phase! In some instances nonlinear corrections have been added; and of course computer simulations based on assumed interatomic potentials automatically include such nonlinear and gradient corrections to linear response. But even then in many cases the host crystal structure is not allowed to change in the simulation.

However, we are explicitly interested in cases where structural transformations are of the essence. In this situation the representation of the host crystal must explicitly contain the possibility of multiphase states. Thus, the standard continuum or Kanzaki methods are intrinsically unsuited for the problem of a defect in a potentially transformable host. And that is the point: the nonlinear, nonlocal Landau-Ginzburg (LG) method provides a representation of the displacive response of the host crystal which is in fact general enough to at least model the effects of localized defects or applied stresses. Furthermore, the particular form of the LG representation of the unperturbed crystal ought to realistically model the behavior of the system under consideration (e.g. not a simple Ising model for a crystal whichmakes first order transitions). With these introductory remarks for perspective we proceed with two examples: point defects, precursors, and martensite transformation processes.

We take it as a given that all real martensitic transformations are controlled by defects. Considering the many kinds of martensites it is not surprising that there have been literally hundreds of studies of different systems, and a long history of models for the nucleation of transformations by defects. This history has been reviewed in depth by Clapp [22]; and recently [19] he has employed the LG/SG framework to model the nucleation of the transformation by a point defect. Roitburd [23] did extensive early work on this topic. Cao, Barsch, Gooding, and the author [24] have applied the LG method in a more specific manner. Clapp's model with a negative SG coefficient produces a description for nucleation below the nominal bulk transition temperature (i.e. undercooled); Cao et.al. show that by contrast, for a material with positive strain gradient coefficient it is possible in principle to have localized transformations above the bulk transformation temperature i.e. the first-order analog of the "localized soft mode" (second order) model due to Clapp (1973). As temperature decreases these islands grow and coalesce, thus a model for athermal martensite.It is proposed that that model is appropriate to many of the shape memory alloys.

In the presence of a defect or applied stress the LG free energy can be written

$$
\Phi_{\text {tot }}=\Phi_{\mathrm{L}}+\Phi_{\mathrm{SG}}+\Phi_{\text {pert }}
$$

where the notation is as previous, and a perturbation term has been added. Following Cao, et. al. [24] for a pedagogical (perhaps hi- $\mathrm{T}_{\mathrm{c}}$ ) tetragonal-rhomobhedral transition model the effect of a homogeneous applied stress on the free energy (the SG term does not enter) is shown in Fig.9 (next page) for different values of the stress $\sigma$ for a reduced temperature $\tau=1.3781$, above the transition condition 1.00 . The interpretation is straightforward; one sees that first, one variant of the rhombohedral phase is preferred over the other except for zero applied stress, second, that with enough applied stress the parent phase transforms into one variant. In this case (dimensionless units) the threshold transformation stress is 0.2 , at which a first order transformation takes place from the elastically strained parent. At still higher stress the parent would become elastically unstable and transform without the necessity of nucleation, but as we shall see next that condition is not relevant to the transformation when non-uniform stress, e.g. due to point defects, is present.

The next step is from a uniform sttress to a localized stress; we can model a point defect, for example, by a spatially localized stress $\sigma=\sigma_{0} /\left[1+\left(\xi / \xi_{0}\right)^{2}\right]$ where $\xi, \xi_{0}$ are distance from the center of the defect, and range of the localized stress, repectively. The strain vs. distance profile is then as shown in Fig. 10, where the dimensionless temperature $\tau$ is the parameter. Now, quite unlike the effect of a defect in a non-transforming crystal, a remarkable thing happens! With a given defect "strength" $\sigma_{0}$, for temperatures much greater than the bulk $M_{S}$ the strain around the defect is like the conventional elastic distortion; as temperature is lowered, though still above $\mathbf{M}_{\mathbf{S}}$, there is a threshold temperature at which the conventional elastic solution becomes unstable against forming an elastically stable, static, localized transformed region of finite spatial extent. As the temperature is lowered still further this region expands. A diffraction study would show a mixture of the austeniteand martensite phases. With a distribution of defect strengths one can model the athermal formation of martensite, where the amount transformed increases as $\mathrm{T}$ is lowered in the parent phase setting off more and more localized transformations. There is 
one more point to emphasize; these transformations are definitely not due to localized soft modes; both within and around the localized transformed region the lattice is elastically stable. There are a number of additional insights gained from these models and [24] should be consulted for details.

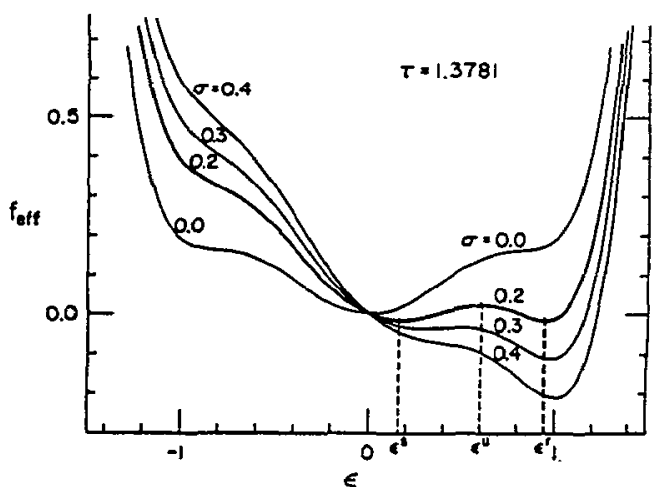

Figure 9. Plot of the effective free-energy density under Figure 10 different homogeneous external stress $\sigma$ for a given temperature $\tau=1.3781$, which is the transition temperature for $\sigma=0.2$. Here $\epsilon^{t}$ and $\epsilon^{\prime}$ represent the strain values for the distorted high: temperature and low-temperature phases, respectively.

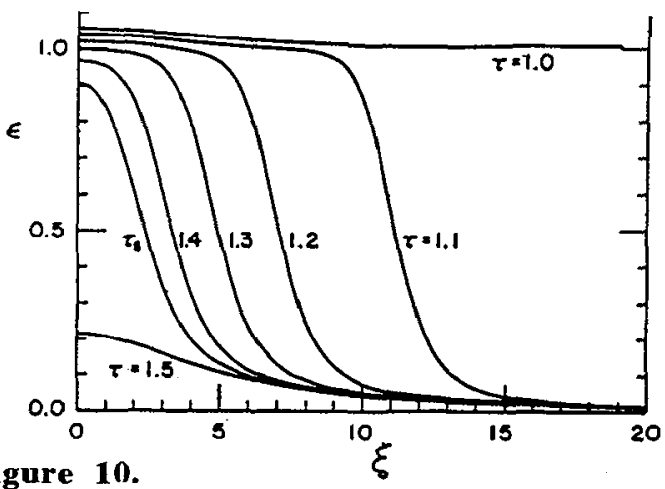

Strain profiles for different temperatures under the stress field $\sigma=\sigma_{0} /\left[1+\left(\xi / \xi_{0}\right)^{2}\right]$ with $\sigma_{0}=0.3, \xi_{0}=5$. Here $\tau_{s}=1.4524$ is the local martensite formation temperature. For $\tau>\tau$, there is only pretransformed distortion of the high. temperature phase as shown by the curve $t=1.5$.

The next type of perturbation, beyond uniform and point localized stress, is the possibility of a spatially extended type of disorder. An inescapable type of spatial disorder is found in the composition variation in space of a disordered, non-stoichimetric alloy which are simply unavoidable. We have shown that the LG model is capable of explaining the tweed precursor structure found over wide ranges for temperatures well above $M_{s}$. The study of tweed, pioneered by Tanner [25], has been one of the more fascinating recent challenges, not only in itself, but for what it might tell us about the underlying transformation processes.we can give only a very brief summary of the main features of the treatment of this problem and key results. The most extensive summary of the work is contained in the Cornell thesis of Dr. Sivan Kartha (1994), and the principal results are in press [26].; an initial report is given in [27]. Here we discuss only a static equilibrium form of tweed which is intrinsic to alloys. A number of other forms of tweed modulation can occur if the elastic distortion is coupled to another degree of freedom, e.g. an order disorder transition [28], and is usually transitional in nature. We indicate the nature of the results here simply by figures. First, a model free energy for a square-rectngular transition is shown in Fig.11 below.

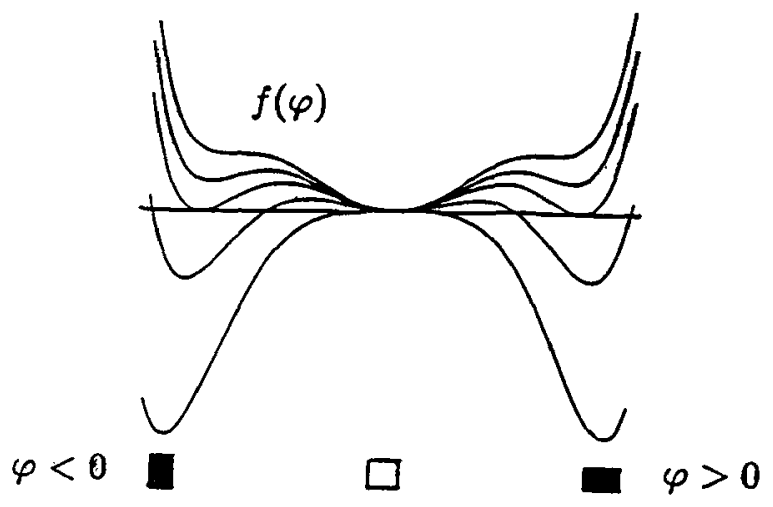

Figure 11. Free energy for square-rectangular transition; various temperatures above and below $\mathbf{M}_{\mathbf{S}}$. 
The main ideas in carrying out an analysis for this case are as follows: First, relate the local composition variation to the free energy; to do this note that there is a tremendous sensitivity of $\mathrm{M}_{S}$ to composition in most SM alloys. We accept this as experimental fact. This is put into the formalism by making $\alpha=\alpha(x, y)$ in the free energy, a spatially random perturbation. As a result, as one moves through the crystal, even at uniform temperature, in a local sense even if $T>M_{S}$, av. some regions will be above/below the transformation condition. The second consideration then enters, which makes this a highly nontrivial problem: the free energy is not an atomistically localized property, there is in fact a coarse graining in its construction which sets a minimum length scale. An extremely fundamental study of this problem was done by Cooke some years ago [29]; the appropriate length is probably of the order of a few lattice constants in most materials with normal phonon spectra. However, there is a third essential ingredient; the distortion of any local region produces long range elastic fields, so would-be locally transformed regions may be inhibited by surrounding regions which are not (nominally) in condition to transform. In essence this introduces another length scale, the "elastic coherence" length; in fact, due to the significant anisotropy of the elastic properties of many of these materials there are several directionally and polarizationally dependent coherence lengths; these are of order of many atomic distances. In fact, taking all these factors together one has a problem in the cooperative behavior of a disordered, locally interacting, but frustrated, system. Such a problem arises in a disordered ferromagnet, and is called the "spin glass" problem; it has been studied extensively in condensed matter physics. Among the main results is that there can be a quite distinct phase from either the ferromagnetic, antiferromagnetic, or paramagnetic phases, called the "spin glass" phase cf. [26,27]. At least in the class of models considered here we relate the spin glass phase to tweed precursors. Here, we can but reproduce two figures from [27], which display various of these features, and leave it to the reader to consult the references for (many) more details.

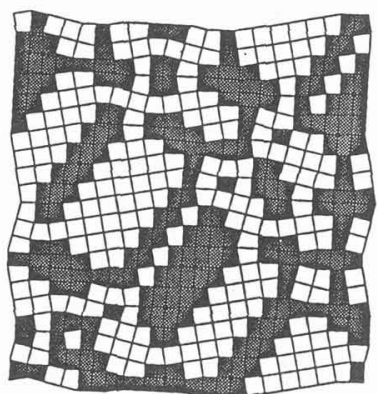

Theoretical tweed. Strained two-dimensional lattice, found by minimizing the elastic free energy for a disordered martensite. The grey regions are mixed between the two rectangular martensitic variants; the white is square austenite.

Figure 12.

Figure13.

\section{Concluding Remarks.}

The purpose of this report was to outline a generic framework into which many martensitic phenomena could be thought of in a moderately unified way, either to think about experiments or for constructing theoretical models. As is generally the case with such generic descriptions they are not in themselves complete enough to discuss specific materials or properties quantitatively. At least, unlike lattice simulations this methodology provides pathways, in principle, between the atomistic physics and continuum theories. It may well be that the application of the methods will be most successful in the shape memory class of materials.

Another line of recent development goes another step beyond the discussion above.Essentially all of the methodology here is only applicable to the reversible elastic aspects of the transformation process. Yet it is also clear that hysterisis, training, and plastic behavior can be strongly present. Those should be the topics for a future conference; there are many new ongoing studies currently.

$I$ am indebted to the organizers and sponsors of this meeting and their hospitality at the University of Barcelona, and to many, many colleagues for their support and stimulus over the past decade, particularly to Gerhard Barsch and Bob Gooding. 


\section{REFERENCES}

Work Supported by Materials Sciences Division, U.S. Dept. of Energy.

[1] "Workshop on First Order Displacive Phase Transitions", Eds.Tanner L.E. and Wuttig M., Materials Science and Engineering A 127 (1990) 137-273.

[2] ICOMAT-92. Eds. Wayman C.M.and Perkins J., Monterey Institute of Advnced Studies 1993,Box 4427, Monterey CA 93921 USA. The present paper is based on the two reports by G.R. Barsch and J.A. Krumhansl in that conference proceedings.

[3] "Martensite" Eds. G.B. Olson and W.S. Owen, ASM International, 1992.

[4] "Phase Transitions in Ferroelastic and Co-elastic Crystals", Salje E.K.H., Cambridge 1993.

[5] See Ref.[2]. Kohn R.V. and Muller S., 77-82; Ball J.M. and James R.D., 6576.

[6] "Shape Memory Alloys" Wayman C.M.,i n Bulletin of the Materials Research Society, April 1993, 49-56.

[7] Heuer A.H.et. al.:J. Am. Ceram. Soc. 63 (1980) 241-248; Acta.Metall 33 (1985) 2101-2112; J. Am. Ceram. Soc. 76 (1993) 1025-1030 . Budiansky

B.and Truskinovsky, J. Mech. Phys. Solids 41 (1993) 1445-1459 .

[8] Toledano T.C. and Toledano P. Phys. Rev. B 21 (1980) 1139-1151; also "The

Landau Theory of Phase Transitions" World Scientific, Singapore 1987.

[9] Horovitz B., Gooding R.J., and Krumhansl J. A. Phys. Rev. Lett . 62 (1989) 843.

[10] Lindgord, P.A. and Mauritsen O. G. Phys. Rev. Lett. 57 (1986) 2458-2461.

[11] Krumhansl J. A. Solid State Comm. 84 (1992) 251-54.

[12] Toupin R. A. Arch. Rational Mec. Anal. 11 (1962) 385-421; 17 (1964) 85-108.

[13] Frank, F. C. Disc. Faraday Soc. 75 (1958) 19-32.

[14] "Ferroelectrics and Related Materials" Lines M.E. and Glass A.M., Chap. 13 Oxford, 1977

[15] "Structural Phase Transitions" Bruce A.D. and Cowley R.A., Taylor and Francis, 1981.

[16] Sato M., Grier B.H., Shapiro S.M., Miyajima H., J. Phys.F: Met. Phys. 12 (1982) 2117- 2125 .

[17] Finlayson T.R., Metall. Trans. 19 A (1988) 185-192; and Smith H. G. ibid.193198.

[18] Smith H. G., Berliner R., Trivissano J., Phys. Rev. B49 (1994) 8547-51.

[19] Clapp P.C. Physica D 66 (1993) 26-34.

[20] Olson G.B. and Cohen M. J. Phys. C. 45 (1982) C475

[21] Barsch G.R. and Krumhansl J.A., Ref. 2 above, 53-64; Metall. Trans. 19A (1988) $761-775$.

[22] Clapp P.C., Ref 1, 189-195.

[23] Roitburd A.L.Solid State Physics 33 (1978) 317-390, and earlier references there.

[24] Cao W. et. al. Phys. Rev. B41 (1990) 11319-11326; B42 (1990) 4334-4340;

B42 (1990) 6396-6401.

[25] Tanner L.E. Philos. Mag. 14 (1966), and Ref. 1, 205-213.

[26] Kartha S., Sethna J. P., Krumhansl J. A., and Wickham L. K. Phys. Rev. B in press.

[27] Kartha S. et al., Phys. Rev. Lett. 67 (1991) 3630-3633.

[28] Salje E. and Parlinski K., Supercon. Science and Technology 4 (1991) 93; with V. Heine J. Phys. Condens. Matter 5 (1993) 497. Semenovskaya S. and Khachaturyn A.G. Phys. Rev. Lett. 67 (1991) 2223-2226.

[29] Cook H.E., Acta. Metall. 23 (1975) 1027; loc.cit.1041. 